Cite this: Metallomics, 2013, 5, 745

Received 15th October 2012, Accepted 29th April 2013

DOI: $10.1039 / c 3 m t 20201 a$

www.rsc.org/metallomics

\section{The G-M-N motif determines ion selectivity in the yeast magnesium channel Mrs2p}

\author{
Gerhard Sponder, $\uparrow^{\mathrm{ab}}$ Soňa Svidová, $\uparrow^{\mathrm{a}}$ Muhammad Bashir Khan, ${ }^{\mathrm{c}}$ Martin Kolisek, ${ }^{\mathrm{b}}$ \\ Rudolf J. Schweyen, $\ddagger^{\mathrm{a}}$ Oliviero Carugo ${ }^{\mathrm{cd}}$ and Kristina Djinović-Carugo*ce
}

\begin{abstract}
The highly conserved G-M-N motif of the CorA-Mrs2-Alr1 family of $\mathrm{Mg}^{2+}$ channels has been shown to be essential for $\mathrm{Mg}^{2+}$ transport. We performed random mutagenesis of the G-M-N sequence of Saccharomyces cerevisiae Mrs $2 \mathrm{p}$ in an unbiased genetic screen. A large number of mutants still capable of $\mathrm{Mg}^{2+}$ influx, albeit below the wild-type level, were generated. Growth complementation assays, performed in media supplemented with $\mathrm{Ca}^{2+}$ or $\mathrm{Co}^{2+}$ or $\mathrm{Mn}^{2+}$ or $\mathrm{Zn}^{2+}$ at varying concentrations, lead to identification of mutants with reduced growth in the presence of $\mathrm{Mn}^{2+}$ and $\mathrm{Zn}^{2+}$. We hereby conclude that (1) at least two, but predominantly all three amino acids of the G-M-N motif must be replaced by certain combinations of other amino acids to remain functional, (2) replacement of any single amino acid within the G-M-N motif always impairs the function of Mrs2p, and (3) we show that the G-M-N motif determines ion selectivity, likely in concurrence with the negatively charged loop at the entrance of the channel thereby forming the Mrs2p selectivity filter.
\end{abstract}

\section{Introduction}

Magnesium is required for numerous cellular processes involved in cell architecture, its growth, energy metabolism and replication. ${ }^{1-5}$ Maintenance of $\mathrm{Mg}^{2+}$ homeostasis is therefore critical for cell viability. Since cellular membranes are impermeable to $\mathrm{Mg}^{2+}$, involvement of various $\mathrm{Mg}^{2+}$-transport mechanisms is required to mediate $\mathrm{Mg}^{2+}$ influx or efflux via plasmalemma and biomembranes forming subcellular compartments.

Members of the large, heterogeneous CorA/Mrs2/Alr1 protein superfamily are found in prokaryotes and in both lower and higher eukaryotes (including regnum of plants). In bacteria (Salmonella enterica ser. typhimurium), yeast (Saccharomyces cerevisiae) and mitochondria of higher eukaryotes they were characterised as being the high-affinity $\mathrm{Mg}^{2+}$ uptake systems. They enable growth of bacteria and yeast in media provided even with very low $\mathrm{Mg}^{2+}$

\footnotetext{
${ }^{a}$ Department of Microbiology, Immunobiology, Genetics, Max F. Perutz Laboratories, University of Vienna, Vienna, Austria

${ }^{b}$ Institute of Veterinary-Physiology, Freie Universität Berlin, Berlin, Germany

${ }^{c}$ Department of Structural and Computational Biology, Max F. Perutz Laboratories, University of Vienna, Campus Vienna Biocenter 5, A-1030 Vienna, Austria. E-mail: kristina.djinovic@univie.ac.at; Fax: +43-1-4277-9522; Tel: $+43-1-4277-52203 / 52201$

${ }^{d}$ Department of General Chemistry, University of Pavia, Pavia, Italy

${ }^{e}$ Department of Biochemistry, Faculty of Chemistry and Chemical Technology, University of Ljubljana, Ljubljana, Slovenia

$\dagger$ These authors contributed equally.

† Deceased on 15th of February 2009.
}

concentrations. $^{6-11}$ Mutants lacking transporters/channels of the CorA/Mrs2/Alr1 superfamily cannot survive without being provided with highly concentrated $\mathrm{Mg}^{2+}$ in the surrounding environment. ${ }^{12-16}$

The MRS2 gene encodes a $54 \mathrm{kDa}$ integral protein of the inner mitochondrial membrane (Mrs2p). Yeast cells lacking $M R S 2$ are respiratory deficient and therefore exhibit a growth defect on non-fermentable substrates ("petite phenotype"). ${ }^{12-16}$ Besides Mrs2p, S. cerevisiae expresses a homologous protein known as Mfm1p/Lpe10p, essential for magnesium homeostasis and group II intron splicing in yeast. ${ }^{14}$ Deletion of MFM1 also results in a "petite phenotype" of the mitochondrial membrane potential $(\Delta \Psi) \cdot{ }^{18-20}$

The three-dimensional crystal structure of the bacterial $\mathrm{Mg}^{2+}$ channel CorA (a distant relative of Mrs2p) has already been solved. ${ }^{18-20}$ Conservation of the primary sequences in the CorA/ Mrs2/Alr1 protein superfamily is in the range of $15-20 \%{ }^{6,8}$ Despite the low primary sequence homology there are several conserved features. These are in particular the two $\alpha$-helices, named "willow helices", in the large N-terminal part and two trans-membrane helices (TM1, TM2) near the C-terminus connected by a short conformationally flexible loop. ${ }^{17}$ The sequence G-M-N, a motif at the end of TM1, and the presence of bulky hydrophobic amino acids in the predicted gate region at the intracellular (intramitochondrial) end of the pore are the only universally conserved features, indicating an essential role of these amino acid residues in the function of CorA/Mrs2/Alr1 proteins. ${ }^{18-20}$ 
By using mag-fura-2 it was shown that Mrs2p behaves as a channel mediating rapid and tightly regulated $\mathrm{Mg}^{2+}$ uptake into mitochondria. ${ }^{9}$ Isolated mitochondria respond to a rise in the external magnesium concentration $\left(\left[\mathrm{Mg}^{2+}\right]_{e}\right)$ with a rapid increase of the mitochondrial free $\mathrm{Mg}^{2+}$ concentration $\left(\left[\mathrm{Mg}^{2+}\right]_{m}\right)$ equivalent to $25 \%$ per s relative to the basal $\left[\mathrm{Mg}^{2+}\right]_{m}$ value. ${ }^{9}$ Utilizing patchclamp, Mrs2p was confirmed to be a $\mathrm{Mg}^{2+}$ channel with a conductance of $\sim 150 \mathrm{pS}^{21}$ Similarly high $\mathrm{Mg}^{2+}$ conductances have been reported by Moomaw and Maguire for CorA (S. typhimurium). ${ }^{22}$ The assumption of a common mechanism of $\mathrm{Mg}^{2+}$ transport for both, Mrs2p and CorA, is further supported by the fact that Mrs2p can functionally substitute for $\operatorname{CorA}^{17}$ and vice versa (this study). Furthermore, $\mathrm{Mg}^{2+}$ transport is in both cases inhibited by cobalt(III)hexammine, a coordinated analogue of the hexahydrated $\mathrm{Mg}^{2+}$ ion. ${ }^{21,23,24}$

Mrs2p has been shown to be permeable also for $\mathrm{Ni}^{2+}$, however, with a 3.5-fold lower conductance $(\sim 45 \mathrm{pS})$ compared to $\mathrm{Mg}^{2+} \cdot{ }^{21}$ It is not permeable for $\mathrm{Ca}^{2+}, \mathrm{Mn}^{2+}$ or $\mathrm{Co}^{2+} \cdot{ }^{21}$ Additionally, suppression of $\mathrm{Mg}^{2+}$ currents in the presence of $\mathrm{Co}^{2+}$ was observed, suggesting $\mathrm{Co}^{2+}$ to interact with the pore. ${ }^{21}$ This is different to $S$. typhimurium CorA and yeast Alr1p for which transport of $\mathrm{Ni}^{2+}$ as well as of $\mathrm{Co}^{2+}$ has been reported. ${ }^{6,23}$

The G-M-N motif has been shown to be critical for the function of CorA and even conservative single amino acid mutations completely abolish $\mathrm{Mg}^{2+}$ transport. ${ }^{25,26}$ This was also confirmed for Mrs2p. ${ }^{9}$ This suggests that this sequence is essential for the function, possibly through suitable positioning of the periplasmic loop implicated in the initial interaction with the hydrated $\mathrm{Mg}^{2+18}$ or/and in assisting in stripping off the hydration shell. ${ }^{22,27,28}$

In previous attempts to obtain a crystal structure of TmCorA, high concentrations of $\mathrm{Mg}^{2+}$ or $\mathrm{Ca}^{2+}$ were used, trapping the protein in its supposable closed state. Very recently, Pfoh et al. have identified a TmCorA mutant that can be crystallized in the presence or absence of $\mathrm{Mg}^{2+} \cdot{ }^{27}$ Furthermore, also the crystal structure of $M$. jannaschii CorA, displaying the extracellular loop, has been published. ${ }^{28}$ Analyses of these two structures have led to new models how conformational changes mediate opening and closing of the channel and how ion selectivity is achieved. ${ }^{27,28}$ In the structure obtained by Pfoh et al. in the presence of $\mathrm{Mg}^{2+}$, one ion was found to be coordinated by $\mathrm{G}$ and $\mathrm{N}$ of the G-M-N motif. The electron density at a distance of $4 \AA$ from the carbonyl group of glycine and the asparagine side chain of the G-M-N motif was interpreted as a bound hydrated $\mathrm{Mg}^{2+}$ ion. ${ }^{27}$ Similarly, a hydrated $\mathrm{Mg}^{2+}$ ion coordinated by the side chain of the asparagine residue of the G-M-N motif was observed in the structure of $M$. jannaschii CorA. ${ }^{28}$ The fact that in both structures a $\mathrm{Mg}^{2+}$ ion is coordinated by the G-M-N motif supports the concept that this sequence is crucial for the initial interaction of the channel with its substrate and for ion selectivity. ${ }^{27,28}$

In order to further investigate the importance and role of the G-M-N motif we performed random PCR mutagenesis on the G-M-N triplet to obtain mutants harbouring all possible amino acid combinations and identified those still capable of transporting $\mathrm{Mg}^{2+}$. The active mutants were further characterized using in vivo and in vitro studies showing that the G-M-N motif can be in part functionally replaced by certain combinations of amino acids. Our results corroborate the notion that this motif plays an important role in ion selectivity.

\section{Results}

\section{Screening of triple G-M-N mutants}

According to the studies of Szegedy and Maguire ${ }^{25}$ and Kolisek and colleagues, ${ }^{9}$ single amino acid substitutions in the G-M-N motif of CorA or Mrs2p are sufficient to abolish $\mathrm{Mg}^{2+}$ transport. Since single conservative mutations in the G-M-N motif are poorly tolerated, we performed a triple site random mutagenesis screen in order to address the question whether any other amino acid combination can substitute for this unique and universally conserved motif.

Since large-scale isolation of mitochondria from yeast is extremely time consuming and thus not suitable for high throughput analyses, we developed a bacteria-based system to screen for functional G-M-N mutants. Based on the ability of Mrs2p to functionally substitute for its bacterial homologue CorA,${ }^{17}$ we assumed that Mrs2p expressed in Salmonella strain MM281 depleted of all major $\mathrm{Mg}^{2+}$ transport systems could complement the $\mathrm{Mg}^{2+}$ transport deficiency. Salmonella strain MM1927, lacking the magnesium uptake systems MgtA and MgtB but over-expressing the $\mathrm{Mg}^{2+}$ channel CorA, considered to be the major $\mathrm{Mg}^{2+}$ uptake system in Salmonella, was used as the positive control. Growth of MM281 cells is only supported at high magnesium concentrations ranging from 10 (as shown in Fig. 1) to $100 \mathrm{mmol} \mathrm{l}^{-1}$. MM1927 and MM281 cells expressing Mrs2p grow equally well without addition of external $\mathrm{MgCl}_{2}$. This clearly proved the ability of Mrs2p to complement the corA $\Delta m g t A \Delta m g t B \Delta$ induced $\mathrm{Mg}^{2+}$ dependent growth defect of strain MM281 and enabled us to investigate de novo generated G-M-N mutants of Mrs2p at the functional level in bacteria.

After transformation of the mutant library into strain MM281, we replica plated the transformants on non- $\mathrm{Mg}^{2+}$. supplemented LB plates containing $0.05 \mathrm{mmol}^{-1}$ IPTG to induce protein expression. These growth-restricting conditions allowed solely mutants still capable of $\mathrm{Mg}^{2+}$-transport to proliferate.

We obtained a considerable amount of G-M-N mutants able to grow without additional $\mathrm{Mg}^{2+}$ supplementation. Out of those 62 mutants were sequenced. In 7 of them the G-M-N motif remained intact, outlining the functional dominance of this sequence. The remaining 55 mutants contained mutations of the G-M-N sequence. In 6 cases we obtained identical mutations in duplicates leaving us with 49 various mutations (Table 1). These 49 mutants were further analyzed using a growth complementation assay on plates supplemented with different IPTG concentrations to investigate how their ability to transport $\mathrm{Mg}^{2+}$ differs from wild-type Mrs2p. The assays were scored with symbols from "++++", for cells able to grow at each of the respective dilution steps to "-" meaning no growth (Table 1).

Bacterial cells transformed with wild-type MRS2 were able to grow well on plates supplemented with only $0.03 \mathrm{mmol} \mathrm{l}^{-1}$ 


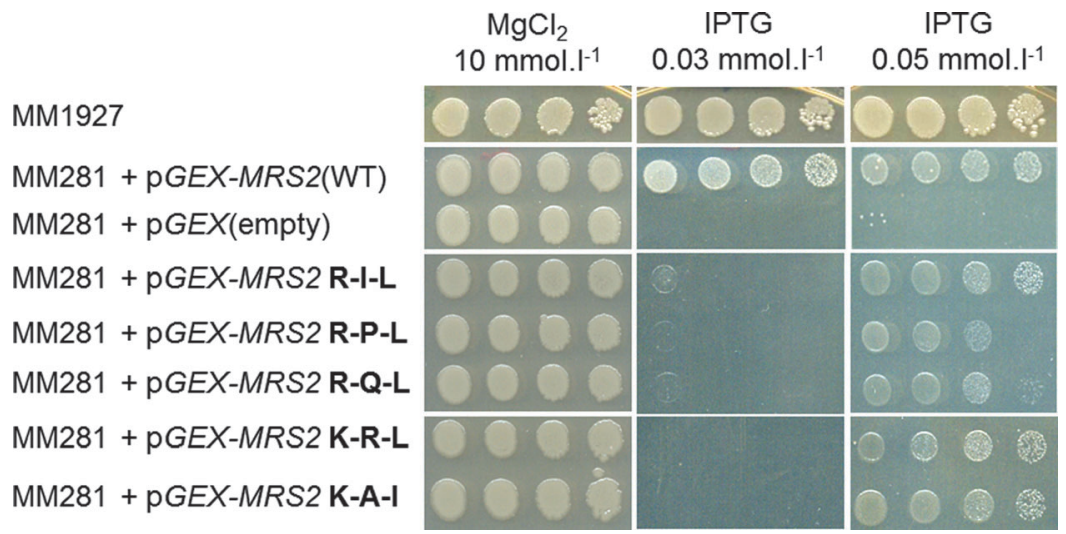

Fig. 1 Representative examples of the growth complementation of the MM281 mutant strain by Mrs $2 p$ and its selected G-M-N mutants. Over-night cultures of MM1927 and MM281 were transformed with plasmids indicated, serially diluted and spotted on LB medium plates supplemented with 10 mmol $\mathrm{I}^{-1}$ magnesium chloride or 0.03 and $0.05 \mathrm{mmol} \mathrm{I}^{-1}$ IPTG and incubated at $37^{\circ} \mathrm{C}$ for 24 hours.

IPTG (Fig. 1). In contrast, the analyzed mutants exhibited only poor growth at this IPTG concentration or did not grow at all (examples are shown in Fig. 1). With increased IPTG concentrations $\left(0.035-0.05 \mathrm{mmol} \mathrm{l}^{-1}\right)$ viability and growth of most of the mutants improved significantly (Table 1 and Fig. 1). These results showed that several (predominantly) G-M-N triple and some double mutants are still capable of mediating $\mathrm{Mg}^{2+}$-influx albeit at lower efficiency than wild-type Mrs2p. The lower transport activity of the functional mutants is reflected by the higher expression levels needed for growth complementation in most of the mutants.

\section{Sequences of the functional mutants}

The prominent feature of the amino acid sequences of functional mutants is their divergence from the canonical G-M-N motif. A glycine at the first position is observed only twice, a methionine appears twice at the second position, and in only one case there is an asparagine at the third position. The only mutant resembling the wild type protein is characterized by the presence of a G-T-N tripeptide instead of G-M-N.

Interestingly, about $80 \%$ of the functional mutants have a positively charged residue in the G-M-N motif. In $59 \%$ of the cases this occurs at the first position, in $18 \%$ of the cases at the second position, and only in $4 \%$ of the cases at the third position. However, the co-presence of two positively charged amino acids is rather uncommon - only in about $10 \%$ of the cases. There is only one evident correlation between two positions; in $59 \%$ of the cases in which the first position is occupied by a positively charged residue, a hydrophobic amino acid (Val, Ile or Leu) occupies the third position. In contrast, a few negatively charged residues are observed in functional mutants, i.e. only six times in the first position and twice in the other two positions. No other clear trends were observed.

In a previous mutational analysis of the G-M-N motif of S. typhimurium CorA, none, even the most conservative single amino acid substitutions (A-M-N, G-A-N, G-C-N, G-I-N, G-M-A, G-M-L, G-M-Q), were tolerated. ${ }^{25}$ A mutational study of Mrs2p performed by Kolisek and coworkers ${ }^{9}$ also confirmed the importance of the G-M-N motif; mutation to A-M-N reduced $\mathrm{Mg}^{2+}$ uptake to the level of the $m r s 2 \Delta$ mutant. These findings are in agreement with our results, as neither these mutants nor any other single amino acid mutation - with the exception of the rather poorly growing G-T-N mutant - were found amongst the functional mutants, which are notably diverse from the native motif G-M-N.

Therefore, it might be concluded that while single substitutions within the canonical G-M-N motif are evolutionarily not tolerated, multiple adjacent mutations, though unlikely to occur in nature, result in functional molecules.

\section{The effect of mutations of the G-M-N sequence on Mrs2p cation selectivity}

We performed a growth complementation assay on plates containing $0.05 \mathrm{mmol} \mathrm{l}^{-1}$ IPTG and different concentrations of $\mathrm{Ca}^{2+}$ or $\mathrm{Co}^{2+}$ or $\mathrm{Mn}^{2+}$ or $\mathrm{Zn}^{2+}$. These ions are known to be transduced by the yeast plasma membrane $\mathrm{Mg}^{2+}$ channel Alr1p, a homologue of Mrs2p. ${ }^{29}$ We selected 10 mutants for this assay (Tables 2 and 3, Fig. 2), in which we examined if the presence of the aforementioned divalent cations in the growth medium influenced cell growth compared to wild-type Mrs2p.

Seven of the chosen mutants had a positively charged amino acid $(\mathrm{K}, \mathrm{R}, \mathrm{H})$ at the first position and a small, hydrophobic residue at the third position (like approx. $35 \%$ of the mutants of Table 1). The last three mutants did not fit in the pattern "positively charged - X - hydrophobic", and were chosen as representatives of well (R-A-W), medium (R-R-T) and poorly (R-V-H) complementing mutant variants.

In the case of $\mathrm{Ca}^{2+}$ and $\mathrm{Co}^{2+}$ we did not observe any difference (results not shown) in the growth complementation assay compared to non-supplemented plates, suggesting that no influx or blockage of the channel by these ions occurred. In a study employing the patch clamp technique on giant lipid vesicles fused with the inner-mitochondrial membrane, Mrs2p was permeable for $\mathrm{Mg}^{2+}$ and $\mathrm{Ni}^{2+}$ but not for $\mathrm{Ca}^{2+}, \mathrm{Mn}^{2+} \mathrm{Or} \mathrm{Co}^{2+}$. Per contra suppression of $\mathrm{Mg}^{2+}$ currents in the presence of $\mathrm{Co}^{2+}$ was seen. ${ }^{21}$ In our case no effect of $\mathrm{Co}^{2+}$ was observed, both on 
Table 1 S. typhimurium strain MM281 was transformed with pGEX-Mrs2(WT) or pGEX carrying various Mrs2 G-M-N mutants (as indicated), serially diluted and replica plated on plates with increasing IPTG concentrations $\left(0.03-0.05 \mathrm{mmol}^{-1}\right)$. The number of " + " symbols corresponds to the number of dilution steps exhibiting growth

\begin{tabular}{|c|c|c|c|c|c|c|}
\hline \multirow[b]{2}{*}{ No. } & \multirow[b]{2}{*}{ Sequence } & \multicolumn{5}{|c|}{ IPTG $\left(\mathrm{mmol} \mathrm{l}^{-1}\right)$} \\
\hline & & 0.03 & 0.035 & 0.04 & 0.045 & 0.05 \\
\hline 1 & G-M-N & ++++ & ++++ & ++++ & ++++ & ++++ \\
\hline 2 & $\mathrm{R}-\mathrm{F}-\mathrm{V}^{a}$ & + & ++++ & ++++ & ++++ & ++++ \\
\hline 3 & R-I-L & + & ++++ & ++++ & ++++ & ++++ \\
\hline 4 & $\mathrm{R}-\mathrm{M}-\mathrm{V}$ & + & ++++ & ++++ & ++++ & ++++ \\
\hline 5 & R-Q-I & + & ++++ & ++++ & ++++ & ++++ \\
\hline 6 & $\mathrm{R}-\mathrm{Q}-\mathrm{L}^{a}$ & + & ++++ & ++++ & ++++ & ++++ \\
\hline 7 & $\mathrm{R}-\mathrm{V}-\mathrm{L}^{a}$ & + & ++++ & ++++ & ++++ & ++++ \\
\hline 8 & L-R-C & ++ & +++ & +++ & ++++ & ++++ \\
\hline 9 & R-A-W & + & ++++ & +++ & ++++ & ++++ \\
\hline 10 & $\mathrm{R}-\mathrm{V}-\mathrm{M}$ & + & ++++ & +++ & ++++ & ++++ \\
\hline 11 & I-R-I & ++ & +++ & ++ & ++++ & ++++ \\
\hline 12 & K-A-I & - & ++++ & +++ & ++++ & ++++ \\
\hline 13 & K-R-L & - & ++++ & +++ & ++++ & ++++ \\
\hline 14 & R-F-I & + & ++++ & +++ & ++++ & +++ \\
\hline 15 & R-V-I & + & ++++ & +++ & ++++ & +++ \\
\hline 16 & R-N-L & - & +++ & +++ & ++++ & ++++ \\
\hline 17 & R-P-L & + & +++ & +++ & ++++ & +++ \\
\hline 18 & C-F-L & ++ & ++ & ++ & +++ & ++++ \\
\hline 19 & R-G-F & + & +++ & +++ & +++ & +++ \\
\hline 20 & R-S-V & + & +++ & +++ & ++++ & ++ \\
\hline 21 & $\mathrm{R}-\mathrm{C}-\mathrm{V}$ & - & ++ & ++ & ++++ & ++++ \\
\hline 22 & F-R-L ${ }^{a}$ & + & ++ & ++ & +++ & +++ \\
\hline 23 & D-F-G & + & ++ & + & +++ & +++ \\
\hline 24 & K-A-M & - & ++ & ++ & +++ & +++ \\
\hline 25 & R-F-Y & + & ++ & ++ & +++ & ++ \\
\hline 26 & R-R-T & - & ++ & ++ & +++ & +++ \\
\hline 27 & V-R-A & + & ++ & ++ & ++ & +++ \\
\hline 28 & V-R-C & + & ++ & ++ & ++ & +++ \\
\hline 29 & D-F-P ${ }^{a}$ & + & ++ & + & ++ & +++ \\
\hline 30 & E-F-P & + & ++ & ++ & ++ & ++ \\
\hline 31 & $\mathrm{~K}-\mathrm{H}-\mathrm{V}$ & - & ++ & ++ & +++ & ++ \\
\hline 32 & E-Q-V & + & ++ & + & ++ & ++ \\
\hline 33 & G-D-M & + & ++ & + & ++ & ++ \\
\hline 34 & K-Y-I & - & ++ & ++ & ++ & ++ \\
\hline 35 & R-T-Y & - & ++ & ++ & ++ & ++ \\
\hline 36 & E-F-A & + & + & + & ++ & ++ \\
\hline 37 & G-T-N & + & + & + & ++ & ++ \\
\hline 38 & K-M-L & - & ++ & + & ++ & ++ \\
\hline 39 & P-D-L ${ }^{a}$ & - & + & ++ & ++ & ++ \\
\hline 40 & R-F-Q & - & ++ & + & ++ & ++ \\
\hline 41 & $\mathrm{R}-\mathrm{V}-\mathrm{H}$ & - & + & + & +++ & ++ \\
\hline 42 & $\mathrm{R}-\mathrm{Y}-\mathrm{S}$ & - & ++ & ++ & ++ & + \\
\hline 43 & R-F-S & - & + & + & ++ & ++ \\
\hline 44 & T-S-E & + & + & + & + & ++ \\
\hline 45 & E-S-K & - & + & + & ++ & + \\
\hline 46 & F-R-E & - & + & + & ++ & + \\
\hline 47 & K-I-T & - & + & + & ++ & + \\
\hline 48 & $\mathrm{P}-\mathrm{N}-\mathrm{V}$ & - & + & + & + & + \\
\hline 49 & P-R-L & - & + & + & + & + \\
\hline 50 & P-T-L & - & - & - & + & + \\
\hline
\end{tabular}

wild-type Mrs2p and on the investigated mutants. A feasible explanation might be the disparity between the approaches used by us and by Schindl and coworkers. ${ }^{21} \mathrm{Co}^{2+}$ concentrations used in the patch-clamp experiments were significantly higher than concentrations used in our experimental setting. Therefore it is not possible to directly compare the results of the growth complementation assay and the patch-clamp experiments of Schindl and colleagues. ${ }^{21}$
Table 2 S. typhimurium strain MM281 was transformed with pGEX-Mrs2(WT) or pGEX carrying various Mrs2 G-M-N mutants (as indicated), serially diluted and replica plated on plates with 0.05 mmol I-1 IPTG and different $\mathrm{MnCl}_{2}$ concentrations. The number of " + " symbols corresponds to the number of dilution steps exhibiting growth

\begin{tabular}{llll}
\hline & \multicolumn{2}{c}{ Concentration $\mathrm{MnCl}_{2}\left(\mathrm{mmol} \mathrm{l}^{-1}\right)$} & \\
\cline { 2 - 4 } Sequence & 0 & 0.01 & 0.1 \\
\hline G-M-N & ++++ & ++++ & ++++ \\
K-R-L & ++++ & +++ & ++ \\
R-A-W & ++++ & +++ & ++ \\
R-R-T & +++ & +++ & ++ \\
K-A-I & ++++ & ++ & + \\
R-M-V & ++++ & ++ & + \\
R-C-V & ++++ & + & + \\
R-F-V & ++++ & + & + \\
R-Q-L & ++++ & + & + \\
R-V-L & ++++ & + & + \\
R-V-H & ++ & ++ & + \\
& & & + \\
\hline
\end{tabular}

Table 3 S. typhimurium strain MM281 was transformed with pGEX-Mrs2(WT) or pGEX carrying various Mrs2 G-M-N mutants (as indicated), serially diluted and replica plated on plates with $0.05 \mathrm{mmol} \mathrm{I}^{-1}$ IPTG and different $\mathrm{ZnCl}_{2}$ concentrations. The number of " + " symbols corresponds to the number of dilution steps exhibiting growth

\begin{tabular}{llll}
\hline & \multicolumn{2}{l}{ Concentration $\mathrm{ZnCl}_{2}\left(\mathrm{mmol} \mathrm{l}^{-1}\right)$} \\
\cline { 2 - 4 } Sequence & 0 & 0.01 & 0.1 \\
\hline G-M-N & ++++ & ++++ & +++ \\
K-R-L & ++++ & +++ & ++ \\
R-R-T & ++++ & +++ & +++ \\
R-A-W & ++++ & ++ & + \\
K-A-I & ++++ & ++ & + \\
R-C-V & ++++ & ++ & ++ \\
R-F-V & ++++ & ++ & + \\
R-M-V & ++++ & ++ & + \\
R-Q-L & ++++ & ++ & + \\
R-V-L & ++++ & ++ & + \\
R-V-H & +++ & +++ & ++
\end{tabular}

The tested concentrations of $\mathrm{MnCl}_{2}$ reduced growth of all mutants, whereas growth of cells harbouring wild-type MRS2 remained unaffected. The negative effect of $\mathrm{Mn}^{2+}$ increased with rising concentrations (Table 2). Furthermore, the growth defect was differently pronounced in the mutants; the top three least affected are characterized by positively charged residues in the first and the second position (K-R-L; R-R-T), while the two most affected mutants carry Arg at the first, and Leu at the third position (R-Q-L; R-V-L) (Table 2).

The effect of $\mathrm{Zn}^{2+}$ was similar to $\mathrm{Mn}^{2+}$, however the growth defect of the mutants was slightly less pronounced (Table 3). The three least affected and the two most affected mutants were the same as those identified in the $\mathrm{Mn}^{2+}$ assay.

\section{$\mathrm{Mg}^{2+}$ influx into isolated mitochondria}

In order to directly investigate $\mathrm{Mg}^{2+}$ influx into isolated mitochondria of selected mutants we used the $\mathrm{Mg}^{2+}$-sensitive dye mag-fura-2 (Fig. 3). The mutants were selected on the basis of good (R-M-V, R-F-V, R-C-V), medium (R-Q-L) or poor (R-V-H) growth complementation capacity in $S$. typhimurium strain MM281 (Table 1). After addition of $\mathrm{Mg}^{2+}$ to a concentration of 


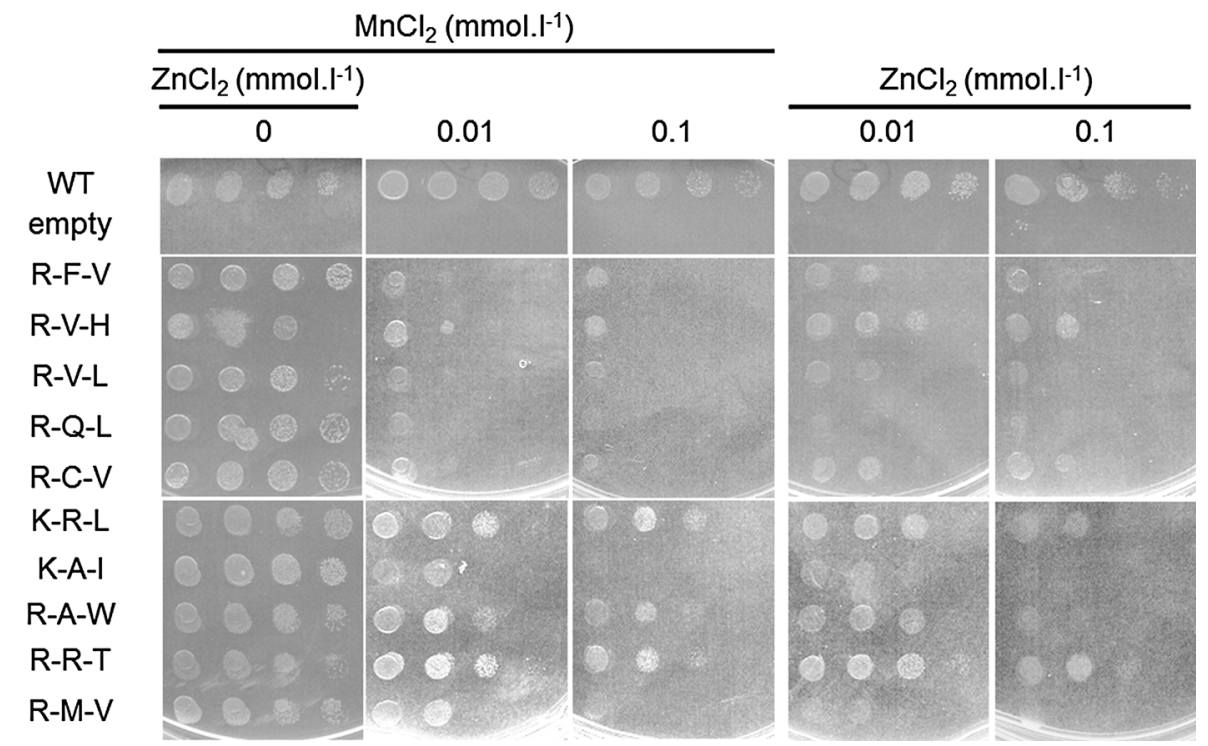

Fig. 2 Influence of $\mathrm{Mn}^{2+}$ and $\mathrm{Zn}^{2+}$ on growth complementation of selected MRS2 mutant variants. S. typhimurium strain MM281 was transformed with pGEX-Mrs2(WT) or pGEX carrying various Mrs2 G-M-N mutants (as indicated), serially diluted and spotted on LB plates supplemented with 0.05 mmol $I^{-1}$ IPTG and different $\mathrm{ZnCl}_{2}$ or $\mathrm{MnCl}_{2}$ concentrations. Plates were incubated at $37^{\circ} \mathrm{C}$ for 24 hours.

$1 \mathrm{mmol} \mathrm{l}^{-1}$ a lack of the characteristic rapid $\mathrm{Mg}^{2+}$ influx ${ }^{9}$ was observed in most mutants, together with significantly lower steady-state $\mathrm{Mg}^{2+}$ levels (Fig. 3). The only exception was the R-Q-L mutant, which lacked rapid $\mathrm{Mg}^{2+}$ influx, but reached a mitochondrial $\mathrm{Mg}^{2+}$ concentration comparable to the wild-type level during the subsequent 100 seconds. After addition of $\mathrm{Mg}^{2+}$ to the final concentration of $3 \mathrm{mmol}^{-1}$, we observed $\mathrm{Mg}^{2+}$ influx in all mutants; however, it did not reach the final steady-state level of wild-type Mrs2p. The differences between mutants were minimal, with the exception of the R-Q-L mutant, which reached almost wild-type $\mathrm{Mg}^{2+}$ levels.

These results indicate that all investigated mutants maintain a certain ability to transport $\mathrm{Mg}^{2+}$ in $S$. typhimurium (Table 1) and mutants R-V-H, R-M-V, R-F-V, R-C-V and R-Q-L also in yeast mitochondria (Fig. 3), albeit in both systems the transport activity was significantly decreased compared to the wild type protein.

\section{Discussion}

The asparagine residues of the G-M-N motif have been previously proposed to block the entrance of the channel in the closed conformation. ${ }^{18,22}$

In the recent study reported by Pfoh et al. it has been suggested that transition of the channel from the closed to the open conformation is mediated by radial and lateral tilts of the protomers that eventually lead to an asymmetric conformation with a bending of the long helix 7 forming the pore.

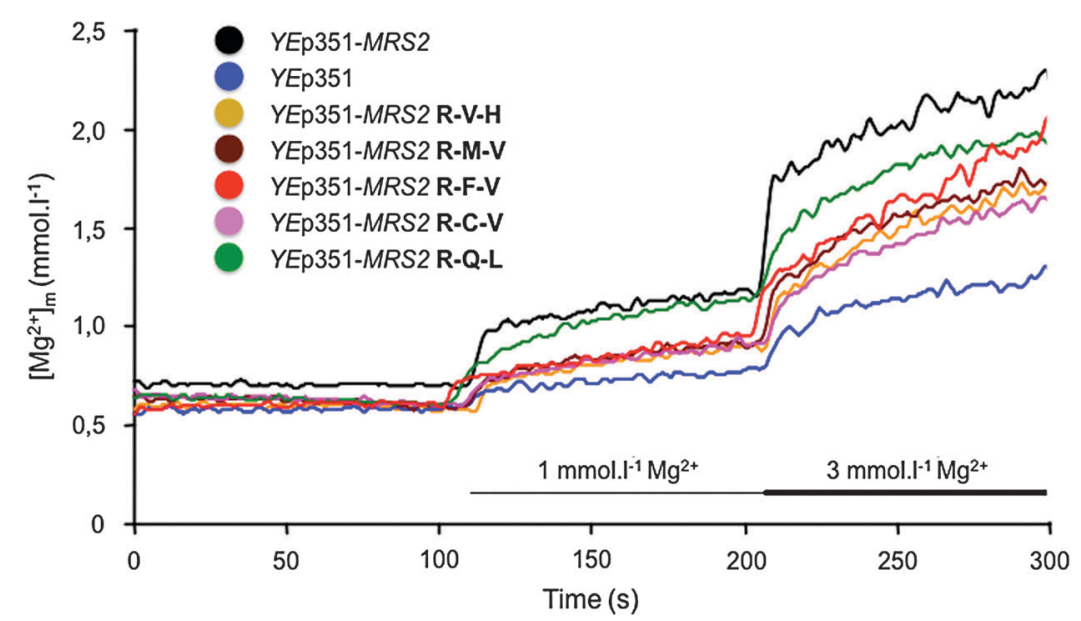

Fig. 3 Representative recordings of $\left[\mathrm{Mg}^{2+}\right]_{m}$ in mitochondria carrying WT and mutated Mrs $2 p$ upon addition of $\mathrm{Mg}^{2+}$ to the measuring buffer. S. cerevisiae strain DBY747 mrs2 4 was transformed with the indicated plasmids and mitochondria were isolated. The representative recordings show changes in matrix Mg ${ }^{2+}$ concentration $\left(\left[\mathrm{Mg}^{2+}\right]_{m}\right.$ ) monitored over 300 seconds. $\mathrm{MgCl}_{2}$ was added in step-wise manner to final $\left[\mathrm{Mg}^{2+}\right]_{e}$ of 1 and 3 mmol ${ }^{-1}$, respectively (as it is indicated). 
Moreover, this bending motion was suggested to pull away one of the periplasmic loops at the mouth of the pore rendering the G-M-N motif accessible for ion coordination. ${ }^{27}$

In the model based on the structure of $M$. jannaschii CorA Guskov et al. proposed that opening of the channel is mediated by a counterclockwise turn of helix 6 upon loss of $\mathrm{Mg}^{2+}$ ions from the intracellular $\mathrm{Mg}^{2+}$ binding sites. This movement eventually replaces hydrophobic residues in the pore with polar residues resulting in a hydrophilic ion permeation pathway. Interestingly, this proposed mechanism does not involve any structural rearrangements of the loop. ${ }^{28}$

Taken together, the loop connecting TM1 and TM2 with its G-M-N motif appears to form the initial interaction site for hydrated $\mathrm{Mg}^{2+}$ and likely participates in the partial dehydration process of the ion required prior to its entrance into the pore of the channel. ${ }^{22,27,28}$ The high conductance of Mrs2 $\mathrm{p}^{21}$ and $\operatorname{CorA}^{22}$ channels was proposed to be based on a mechanism which involves electrostatic interactions of the loop residues with the hydration shell of $\mathrm{Mg}^{2+}$ and not with the ion itself. ${ }^{22}$ This notion is supported by the two recently published structures in which a partially hydrated $\mathrm{Mg}^{2+}$ ion was observed in the loop region at the level of the G-M-N motif with a distance of $4 \AA$ from the coordinating amino acid residues. ${ }^{27,28}$

Our study on the G-M-N motif identified viable triple mutants hosting a positively charged residue primarily on the first but also on the third position. At a first glance, the presence of positively charged residues in functional mutants might seem counterintuitive as these mutations could in fact hinder the transport of $\mathrm{Mg}^{2+}$ ions by electrostatic repulsion. However, it is structurally unfeasible for all three amino acid residues of the motif to be in direct contact with the ion. ${ }^{26-28}$ It is plausible to envisage that these residues form a structural motif critical for ion uptake, which can be partially accomplished by different amino acid combinations, eventually leading to a functionally equivalent structure. Furthermore, it is possible to hypothesize that backbone interactions with the ion cargo are more important than side-chain interactions, which would explain the broad variation of amino acids capable of sustaining selectivity and transport.

In order to assess the impact of mutations in the G-M-N motif on the selectivity of Mrs2p, we performed growth complementation assays on plates supplemented with different cations $\left(\mathrm{Ca}^{2+}, \mathrm{Co}^{2+}, \mathrm{Mn}^{2+}\right.$ and $\left.\mathrm{Zn}^{2+}\right)$. Our results show that mutations in the G-M-N motif lead to reduced growth of the cells in the presence of $\mathrm{Mn}^{2+}$ and $\mathrm{Zn}^{2+}$ while $\mathrm{Ca}^{2+}$ and $\mathrm{Co}^{2+}$ did not influence their viability. This can take place via two possible competitive mechanisms: (1) $\mathrm{Mn}^{2+}$ and $\mathrm{Zn}^{2+}$ are transported through the pore and the growth defect is caused by $\mathrm{Mn}^{2+} / \mathrm{Zn}^{2+}$ intoxication, or (2) $\mathrm{Mn}^{2+}$ and $\mathrm{Zn}^{2+}$ ions are trapped and block the channel for $\mathrm{Mg}^{2+}$ transport causing in this way the growth defect by $\mathrm{Mg}^{2+}$ deficiency. Further studies are needed to clarify the effects of an alteration of the G-M-N sequence on the structural characteristics in this region of the channel.

For CorA ${ }^{25,26}$ and Mrs2 $\mathrm{p}^{9}$ proteins it has been shown that even very conservative mutations in this sequence are not tolerated. However, we were able to identify viable mutants with amino acids greatly differing from G-M-N. This suggests that the individual amino acids of this motif are not critical but the structural properties generated collectively by this motif. This can be partially accomplished by amino acid combinations of completely different individual properties. It seems that $\mathrm{G}-\mathrm{M}-\mathrm{N}$ is the best combination nature found to ensure a high selectivity combined with a high transport capacity.

\section{Materials and methods}

\section{Bacterial cells, growth media and genetic procedures}

Escherichia coli DH10B: F-; endA1; recA1; galE15; galK16; nupG; rpsL; $\Delta$ lacX74; Ф80lacZ $\Delta \mathrm{M} 15$; araD139 $\Delta$ (ara,leu)7697; mcrA $\Delta$ (mrr-hsdRMS-mcrBC) $\lambda$; Salmonella enterica serovar Typhimurium transmitter strain LB5010: metA22; metE551; ilv-452; leu-3121; trpC2; xyl-404; galE856; hsdL6; hsdSA29; hsdSB121; rpsL120; H1-b H2-e n x flaA66 nml (-) Fel-2(-); $\mathrm{Mg}^{2+}$ dependent Salmonella enterica serovar Typhimurium strain MM281: DEL485(leuBCD); mgtB::MudJ; mgtA21::MudJ; corA45::mudJ; zjh1628::Tn10(cam); $\mathrm{Cam}^{\mathrm{R}}, \mathrm{Kan}^{\mathrm{R}}$ (it lacks all major magnesium transport systems and therefore requires medium containing $\mathrm{Mg}^{2+}$ concentrations in the millimolar range); Salmonella enterica serovar Typhimurium strain MM1927: DEL485(leuBCD); mgtB::MudJ; mgtA21::MudJ; corA45:: mudJ; zjh1628::Tn10(cam); $\operatorname{Cam}^{\mathrm{R}}$, $\operatorname{Kan}^{\mathrm{R}}$, pALTER-CorA $\left(\mathrm{Amp}^{\mathrm{R}}\right)$.

Strains were grown in LB medium (1\% tryptone, $0.5 \%$ yeast extract, $1 \% \mathrm{NaCl})$ with ampicillin $\left(100 \mu \mathrm{g} \mathrm{ml} \mathrm{ml}^{-1}\right)$. MM281 required addition of $10 \mathrm{mmol}{ }^{-1} \mathrm{MgCl}_{2}$. LB plates contained 2\% Difco Agar Noble minimizing possible $\mathrm{Mg}$ contamination.

\section{Yeast cells, growth media and genetic procedures}

The $S$. cerevisiae strain DBY747 mrs2 4 deletion strain (DBY mrs2-1, short) has been described previously. ${ }^{12-14}$ Yeast cells were grown in rich medium (YPD; $1 \%$ yeast extract, $2 \%$ peptone and $2 \%$ glucose). ${ }^{11}$

\section{Plasmid constructs}

The construct YEp351-MRS2-HA was described previously by Bui and coworkers. ${ }^{17}$ For cloning of MRS 2 into the vector pGEX$3 \mathrm{X}$ with an IPTG-inducible promoter the primers M2GEXfw: 5'-CGCGGATCCCCAATCGGCGTCTCCTGG-3' and MRS2HiXrev: 5'-TGCTCTAGATCAATGgTGATGGTGATGG-3' were used. The resulting PCR fragment was cloned into the vector via BamHI and XbaI restriction sites.

\section{Random PCR mutagenesis}

In order to introduce various amino acid substitutions in Mrs2p, overlap extension PCR according to Pogulis and coworkers ${ }^{30}$ was used. No additional mutations were found by sequencing. Random mutagenesis of the G-M-N motif of MRS2 was performed with the mutagenic forward primer $5^{\prime}$-GCATCTGTTCTGCCGGCGTTCTATN NNNNNNNNTTAAAGAATTTCATCGAGGAGAGTG- $3^{\prime}$ and the reverse primer $5^{\prime}$-CACTCTCCTCGATGAAATTCTTTAANNNNNNNNNATAG AACGCCGGCAGAACAGATGC-3' according to standard protocols. PCR products were digested with XhoI and EcoRI and cloned into an XhoI and EcoRI digested pGEX-MRS2 construct. For transformation into DH10B a standard calcium chloride method was used. 
Correctly ligated constructs were identified by deletion of the BsmI restriction site of $M R S 2$, resulting in a silent mutation from an adenine to a guanine.

\section{Identification of tolerated substitutions}

A total of 45600 constructs were pooled and transformed into S. typhimurium strain LB5010. A total of 46848 constructs were pooled and transformed into $S$. typhimurium strain MM281, plated on LB plates supplemented with $10 \mathrm{mmol} \mathrm{l}^{-1} \mathrm{MgCl}_{2}$ and replica plated on LB plates containing $0.05 \mathrm{mmol}^{-1}$ IPTG to induce protein expression. Expression of the mutant Mrs2p variants was controlled by Western blot to avoid misinterpretations due to strongly differing expression levels. Forty nine mutants able to grow on this medium were sequenced. No additional mutations were found by sequencing.

\section{Serial dilutions}

For serial dilutions on plates, cells were grown in liquid LB medium containing $10 \mathrm{mmol} \mathrm{l}^{-1} \mathrm{MgCl}_{2}$ at $37{ }^{\circ} \mathrm{C}$ overnight, washed twice with LB medium, adjusted to an $A_{600}$ of 1 and diluted to an $A_{600} 0.1,0.01$ and 0.001 . Serial dilutions were spotted onto LB medium plates containing different concentrations of $\mathrm{MgCl}_{2}$, IPTG, $\mathrm{MnCl}_{2}$ or $\mathrm{ZnCl}_{2}$ and incubated for $24 \mathrm{~h}$.

Isolation of mitochondria and measurement of changes in the intramitochondrial $\mathrm{Mg}^{2+}$ concentrations by mag-fura 2 assisted spectrofluorimetry

Isolation of mitochondria by differential centrifugation and ratiometric determination of intramitochondrial $\mathrm{Mg}^{2+}$ concentrations $\left(\left[\mathrm{Mg}^{2+}\right]_{m}\right)$ dependent on various external concentrations $\left(\left[\mathrm{Mg}^{2+}\right]_{e}\right)$ was performed as described previously. ${ }^{9}$

\section{Conclusions}

In summary we conclude that the G-M-N motif, despite its high degree of conservation, can be functionally replaced by certain combination(s) of amino acid residues. Most frequently a positively charged residue in the first and a hydrophobic residue in the third position were found in functional mutants. Our studies suggest that the G-M-N motif plays a role in ion selectivity, being therefore part of the selectivity filter together with the flanking negatively charged loop, at the entrance of the Mrs2p channel. The eminent involvement of the G-M-N motif in ion selectivity might be the molecular basis for its universal conservation throughout the phyla.

\section{Authors' contributions}

GS, SS and MBK conducted experiments; RS, GS and KDC designed experiments; RS, GS, SS, MBK, MK, OC and KDC evaluated data; MK, GS, SS, OC and KDC wrote the manuscript.

\section{Acknowledgements}

We thank Michael Maguire (Case Western Reserve University) for providing the Salmonella typhimurium strains MM281 and
MM1927. We thank Elisabeth Froschauer (Max F. Perutz Laboratories, Univ. Vienna, Austria), Rainer Schindl, Julian Weghuber and Christoph Romanin (all Univ. Linz, Austria) for critical comments on the manuscript. Dr. Theresa Jones is gratefully acknowledged for the linguistic corrections and text editing. MBK, GS, SS were recipients of a PhD fellowship from Austrian Science Fund FWF (P20141) and from Vienna Science and Technology Fund WWTF (LS05021). MBK was partially supported by the University of Vienna funds. The BIN-III initiative of the Austrian GEN-AU is acknowledged for financial support.

\section{References}

1 J. A. Cowan, Inorg. Chem., 1991, 30, 2740-2747.

2 J. A. Cowan, Chem. Rev., 1998, 98, 1067-1088.

3 J. A. Cowan, T. Ohyama, K. Howard, J. W. Rausch, S. M. Cowan and S. F. Le Grice, JBIC, J. Biol. Inorg. Chem., 2000, 5, 67-74.

4 C. E. Dann, 3rd, C. A. Wakeman, C. L. Sieling, S. C. Baker, I. Irnov and W. C. Winkler, Cell, 2007, 130, 878-892.

5 G. J. Quigley, M. M. Teeter and A. Rich, Proc. Natl. Acad. Sci. U. S. A., 1978, 75, 64-68.

6 R. C. Gardner, Curr. Opin. Plant Biol., 2003, 6, 263-267.

7 A. Graschopf, J. A. Stadler, M. K. Hoellerer, S. Eder, M. Sieghardt, S. D. Kohlwein and R. J. Schweyen, J. Biol. Chem., 2001, 276, 16216-16222.

8 V. Knoop, M. Groth-Malonek, M. Gebert, K. Eifler and K. Weyand, Mol. Genet. Genomics, 2005, 274, 205-216.

9 M. Kolisek, G. Zsurka, J. Samaj, J. Weghuber, R. J. Schweyen and M. Schweigel, EMBO J., 2003, 22, 1235-1244.

10 M. Wachek, M. C. Aichinger, J. A. Stadler, R. J. Schweyen and A. Graschopf, FEBS J., 2006, 273, 4236-4249.

11 J. Weghuber, F. Dieterich, E. M. Froschauer, S. Svidova and R. J. Schweyen, FEBS J., 2006, 273, 1198-1209.

12 H. Koll, C. Schmidt, G. Wiesenberger and C. Schmelzer, Curr. Genet., 1987, 12, 503-509.

13 G. Wiesenberger, M. Waldherr and R. J. Schweyen, J. Biol. Chem., 1992, 267, 6963-6969.

14 J. Gregan, D. M. Bui, R. Pillich, M. Fink, G. Zsurka and R. J. Schweyen, Mol. Gen. Genet., 2001, 264, 773-781.

15 J. Gregan, M. Kolisek and R. J. Schweyen, Genes Dev., 2001, 15, 2229-2237.

16 G. Zsurka, J. Gregan and R. J. Schweyen, Genomics, 2001, 72, 158-168.

17 D. M. Bui, J. Gregan, E. Jarosch, A. Ragnini and R. J. Schweyen, J. Biol. Chem., 1999, 274, 20438-20443.

18 V. V. Lunin, E. Dobrovetsky, G. Khutoreskaya, R. Zhang, A. Joachimiak, D. A. Doyle, A. Bochkarev, M. E. Maguire, A. M. Edwards and C. M. Koth, Nature, 2006, 440, 833-837. 19 S. Eshaghi, D. Niegowski, A. Kohl, D. Martinez Molina, S. A. Lesley and P. Nordlund, Science, 2006, 313, 354-357. 20 J. Payandeh and E. F. Pai, EMBO J., 2006, 25, 3762-3773. 21 R. Schindl, J. Weghuber, C. Romanin and R. J. Schweyen, Biophys. J., 2007, 93, 3872-3883. 
22 A. S. Moomaw and M. E. Maguire, Biochemistry, 2010, 49, 5998-6008.

23 R. L. Smith and M. E. Maguire, Mol. Microbiol., 1998, 28, 217-226.

24 L. M. Kucharski, W. J. Lubbe and M. E. Maguire, J. Biol. Chem., 2000, 275, 16767-16773.

25 M. A. Szegedy and M. E. Maguire, J. Biol. Chem., 1999, 274, 36973-36979.

26 J. Payandeh, C. Li, M. Ramjeesingh, E. Poduch, C. E. Bear and E. F. Pai, J. Biol. Chem., 2008, 283, 11721-11733.
27 R. Pfoh, A. Li, N. Chakrabarti, J. Payandeh, R. Pomes and E. F. Pai, Proc. Natl. Acad. Sci. U. S. A., 2012, 109, 18809-18814. 28 A. Guskov, N. Nordin, A. Reynaud, H. Engman, A. K. Lundback, A. J. Jong, T. Cornvik, T. Phua and S. Eshaghi, Proc. Natl. Acad. Sci. U. S. A., 2012, 109, 18459-18464.

29 C. W. MacDiarmid and R. C. Gardner, J. Biol. Chem., 1998, 273, 1727-1732.

30 R. J. Pogulis, A. N. Vallejo and L. R. Pease, Methods Mol. Biol., 1996, 57, 167-176. 